\title{
Unification beyond justification: a strategy for theory development
}

\author{
Molly Kao
}

\begin{abstract}
This paper considers the importance of unification in the context of developing scientific theories. I argue that unifying hypotheses are not valuable simply because they are supported by multiple lines of evidence. Instead, they can be valuable because they guide experimental research in different domains in such a way that the results from those experiments inform the scope of the theory being developed. I support this characterization by appealing to the early development of quantum theory. I then draw some comparisons with discussions of robustness reasoning.
\end{abstract}

Keywords Unification · Theory development · Quantum theory · Robustness reasoning

\section{Introduction}

The idea of unification has a long and varied history in philosophy of science. Consider, for instance, William Whewell's discussion of the Consilience of Inductions, first introduced in his Novum Organon Renovatum as part of his analysis of the structure of scientific reasoning. One important element in the construction of a theory occurs through the "Colligation of Facts", a process he called Induction (Whewell, 1989, p. 138). The three steps of this process are (1) the selection of the idea, (2) construction of the conception and (3) determination of the magnitudes. In contemporary terms, we might think of these steps as analogous to those used when trying to characterize a particular phenomenon mathematically. This process warrants the name 'Induction' because, as Whewell argues, something new is added when such a characterization occurs, namely, previously ascertained facts are linked by a new conception, often a mathematical equation. For instance, multiple observations of the position of Mars are individual facts, which were colligated into the single conception of an elliptical orbit. However, such an Induction refers to merely one class of facts. A Consilience of Inductions occurs when an Induction obtained from one class is found to apply to a different class of facts, even though the two previously seemed to be unrelated. Whewell takes this to be a good indicator of probable truth. He says,

[T] he evidence in favour of our induction is of a much higher and more forcible character when it enables us to explain and determine cases of a kind different from those which were contemplated in the formation of our hypothesis. The instances in which this has occurred, indeed, impress us

I am grateful for the valuable feedback I received from the audience members at the workshop on Exploring Scientific Method at the Munich Center for Mathematical Philosophy in May 2017, as well as the annual 2017 conference of the Canadian Philosophical Association. I would also like to thank Michel Janssen and two anonymous referees for their helpful comments.

Address(es) of author(s) should be given 
with a conviction that the truth of our hypothesis is certain. No accident could give rise to such an extraordinary coincidence $(1989,153$, emphasis in original).

Thus, he argues that when a hypothesis is able to explain or predict facts of a kind that were not used in its generation, the evidence for that hypothesis is stronger than it was before on the grounds that it could not be mere coincidence that two disparate phenomena are explained by the same hypothesis. Instead, it is much more likely that the hypothesis does actually apply in some way to all the phenomena it explains.

There are several historical examples that seem to bear out this inference. For instance, consider Newton's "Moon-test" in his Principia. Having already established that there is some force keeping planets in their orbits around the Sun, and that this force is directed towards the center of the Sun, Newton goes on to argue that a similar force keeps the moon in orbit around the Earth, and that this force is the same force previously recognised as terrestrial gravity. To make this argument, Newton first argues that the force maintaining the moon in its orbit is directed towards the earth. He then assumed a mean Earth-Moon distance of 60 Earth radii. Now, to quote William Harper,

Newton's assumption of 60 terrestrial semidiameters as the lunar distance, together with inversesquare variation, makes the one-minute fall corresponding to the strength of this force at the lunar distance exactly equal to the one-second fall corresponding to the increased strength this force would have at the surface of the Earth (Harper, 2002, 182).

Thus, what we have is a force acting on the Moon directed towards the center of the Earth, whose strength is equal to a force at the surface of the Earth that acts on all objects there, whose magnitude is measured by a seconds pendulum. Newton then refers to his Rules of Reasoning in order to posit that the two forces responsible for the two phenomena are one and the same. We observe here the idea of unification: the force that we observe as the phenomenon of terrestrial gravity is able to account for the observations of the moon's orbit around the Earth, two phenomena which previously seemed unrelated.

While Whewell's term 'consilience' is not widely used, ${ }^{1}$ the idea of unification has reappeared in the literature in several manifestations, where a useful characterization can be given as follows: unification as a feature of scientific theories or hypotheses refers to the ability of said theory or hypothesis to account for multiple phenomena which, prior to the proposal of the unifying hypothesis, appeared to be unrelated. The value of this feature has been analysed in several different ways, which can be grouped roughly into two types. The first is drawn from the work of philosophers such as Philip Kitcher, who appealed to the feature of unification in order to characterize and evaluate scientific explanations (1981; 1989). He argued that science provides good explanations to the extent that its theories are able to account for a wide range of phenomena. Thus, if explanation is taken to be a goal of science, so is unification. The degree of unification of an explanation can thus contribute to the evaluation of its quality, and by extension, the quality of the theory itself.

It is now arguably more common to find unification being discussed in the context of confirmation and justification. This characterization can take one of a few forms, but what these accounts have in common is that they consider the feature to contribute in some way to the evidential support for the unifying hypothesis or theory. One way that this is cashed out is in terms of a common cause, or a type of inference to the best explanation. For example, Forster (1988) has argued that theories that unify phenomena can be understood as cases where causes from different contexts are independently verifying one another, thus gaining credibility. ${ }^{2}$ We also see unification discussed in the context of formal epistemology, where authors such as Myrvold (2003, 2017), McGrew (2003), Helgeson (2013), and Schupbach (2005) explicate the concept using a Bayesian framework to analyse the degree of support it provides to a theory.

Without doubt, I consider these discussions both interesting and useful for our understanding of unification. However, I submit that our focus on these conceptions has caused us to undervalue a crucial aspect

\footnotetext{
1 However, see Forster (2010) for an analysis in contemporary physics specifically using Whewell's notion of consilience.

2 Spohn (1994) has also discussed unification in relation to IBE and common cause.
} 
of unification in scientific inquiry. This is in its use as a strategy for guiding theory pursuit, especially in the early stages of theory development when a fully consistent framework may not yet be available. Due in part to a tendency to consider unification primarily in the context of more developed rather than fledgling theories, its utility as a heuristic is often overlooked. In such cases, the value of a unifying hypothesis is not necessarily that of its ability to explain phenomena, nor that it raises the likelihood that the theory or hypothesis is true. Instead, unifying hypotheses can be valuable because they guide experimental research in different domains in such a way that the results from those experiments contribute to our understanding and formulation of the theory being developed. The unification strategy helps us to pinpoint what must be retained in any subsequent theory by identifying elements that are common to the different areas of inquiry, as well as delineating the domain of applicability of the theory.

We see a hint of this in Michel Janssen's discussion of what he terms COI Stories, an acronym for common origin inferences, which he considers a subspecies of Inferences to the Best Explanation (Janssen, 2002). COIs arise out of what Janssen calls common-origin explanations, which are hypotheses or theories that tie together multiple phenomena. He believes that most COIs will be common-cause inferences, in which some kind of causal structure is posited as being responsible for several phenomena. Of course, the notion of 'causal structure' here is quite broad, possibly referring to some event or substance with causal efficacy, or a causal structure or mechanism broadly construed. The latter could presumably include such things as physical laws described by some mathematical structure, and so does not restrict such an inference to any particular account of causal relations. Janssen provides a detailed reconstruction of several historical COI stories, and shows that this kind of reasoning did contribute to scientists' decisions to accept certain theories. He also concludes that "the reliance on COIs in ... the context of pursuit does not call for any general philosophical justification. The issue of the justification of COI as a pattern of inductive inference only comes up in the context of persuasion," $(2002,513)$. While I am generally in agreement with this statement, I believe it would be helpful to focus more attention on the role of unification in the former context. When we do so, we can see that the idea of a common origin as explicated by Janssen is very similar to that of unification. However, we should characterize its role in the "context of pursuit" not necessarily as an inference to the best explanation, but instead as a heuristic to guide theory development. ${ }^{3}$

My characterization of unification as a guiding strategy for theory construction is similar to the kind of reasoning found in discussions of robustness analysis and indeed, its bears some resemblance to arguments that have been given in that context. In particular, philosophers who discuss robustness often consider the process of developing a theory rather than evaluating it. ${ }^{4}$ However, there is an important difference in our focus. Those who are interested in robustness reasoning tend to be interested in the study and description of a particular system or phenomenon that is represented by multiple models. Robustness reasoning is a way of inferring that some contents of these models are correct, and thus representative of the phenomenon under study. Unification, in my account, is meant to be a heuristic strategy for pursuit that guides research in multiple domains.

The aim of this paper is to show that the trait of unification can be considered in terms of a heuristic strategy rather than a justificatory feature by showing how it was operative in a particular historical example. While there is much to be said on the topic of heuristics, in the context of this paper, I consider a unifying hypothesis to have been used heuristically in the sense that it was used to solve problems in different domains without a clear account of how and why it should be able to do so. While not denying the importance of the justificatory role of unification, the heuristic conception has the advantage of being able to account for the contribution of failed lines of inquiry to scientific theory development. The rest of the paper proceeds as follows. In the next section, I show how a unifying hypothesis was used in the early

\footnotetext{
3 In recent talks, Janssen has characterized the force of a COI not as a legitimate inference to the truth of a theory, but as an indicator that an idea is worth pursuing.

4 See Weisberg (2006), Trizio (2012), Nederbragt (2012), Kuorikoski and Marchionni (2016) and Schupbach (Forthcoming) for examples. Miyake (2015) discusses a related methodological technique of decomposition in the context of theory construction.
} 
developmental stages of quantum theory. In Section 3, I show how an emphasis on the process of theory development rather than theory evaluation can help us to reconceptualize the role that unification can play. This is accomplished by discussing how understanding unification as a tool for theory development helps to actually define the theory under investigation. I then compare this analysis of unification with analyses of robustness reasoning before making some concluding remarks.

\section{Unification as a guide to theory pursuit: the quantum case study}

One of the central assumptions in classical physics is the idea that energy is correctly described in continuous terms. However, in the late nineteenth-century, various experiments and observations began to point to the inadequacy of classical theories in accounting for certain phenomena. Max Planck's introduction of "energy elements" in 1900 as a way to recover the behaviour of blackbody radiation was a groundbreaking step, and was followed by other applications of the idea of quantized, or discretized, amounts of energy by scientists in diverse contexts. However, there was certainly no well-developed, consistent theory that could account for all of these anomalous observations. Indeed, it was this work that led to the eventual articulation of the theory of quantum mechanics, one of physics' most successful theories to date.

One of the facts that drove the development of quantum mechanics was the appearance of the notion of quantization in several physical contexts. Unification appears to have played a broadly justificatory role insofar as quantum theory was supported by the fact that it could account for phenomena in different domains. ${ }^{5}$ However, while such an account might function as a reconstruction of why elements of a quantum theory were justified, in order to gain a better understanding of theory development, we must also consider unification as it was operative historically. I claim that in that context, unification is best considered a strategy for theory pursuit.

Although the idea of energy quantization was appealed to in several domains, these were not simply applications of a single, unambiguous hypothesis: the tentative conjectures were based on quite different assumptions about the physical reasons for quantization in each context. Indeed, it is more accurate to characterize the scientists in question as appealing to various members of a family of quantum conjectures, with the basic unifying hypothesis of energy quantization in the background guiding these applications. In what follows, I give a brief description of some uses of a quantization conjecture between 1900 and 1913, outlining the relevant differences, in order to show that the idea of quantization is best characterized as a successful strategy for guiding work in which the goal was to develop a new theory that could account for observed physical anomalies. For Einstein especially, this heuristic conception played a central role in several theoretical advancements.

It was the investigation of blackbody radiation which first gave rise to the consideration of an explicit quantum conjecture by Planck. ${ }^{6}$ In his description, Planck employed a model of a blackbody as a system of Hertzian resonators - small, nonresistive, oscillating electric circuits. While most of the details are not important here, we can describe a major part of Planck's task as determining the relation between the mean energy and entropy of a single resonator in the system. To do this, he considered a large number $N$ of identical resonators, and took the total entropy of the resonators $S_{N}$ to be $k \log W+$ const, in analogy to the entropy found in kinetic gas theory. In order to find a value for $W$, the probability that the system of resonators possesses energy $U_{N}$, Planck assumed that the energy can be considered in portions of yet undetermined size $\epsilon$. Rather than simply taking the limit as $\epsilon \rightarrow 0$ as one would expect for continuous amounts of energy, he used this discretized consideration of the energy $U_{N}$ to calculate the number of ways the energy elements could be divided between the $N$ resonators, thus yielding a value

\footnotetext{
5 See Kao (2015) for a reconstruction of such an argument.

6 This refers to the spectrum of radiation emitted by an ideal body that perfectly absorbs, then re-emits all radiation incident upon it.
} 
for $W$. Further calculations yielded the result that $\epsilon$ must be proportional to frequency $\nu$, and Planck introduced the constant $h$ to express that proportionality as $\epsilon=h \nu$.

Based on various presentations of his idea of quantization (Planck 1901, 1900), it is possible to attribute two different quantum conjectures to Planck. It is not clear which of these Planck was genuinely committed to, if he indeed had a single conjecture in mind. Both conjectures arise from an interpretation of the "energy element" $\epsilon=h \nu$, and what it might mean for energy of frequency $\nu$ to be considered in amounts of $\epsilon$.

One way to read Planck's quantization condition is to attribute the discontinuous nature of energy to the behaviour of the resonators modeling the blackbody. In this case, one assumes only that we must describe the energy that a single resonator possesses in integral multiples of $h \nu$. This is how historians such as Klein have read Planck, who says for instance "Planck had quantized only the energy of the material oscillators and not the radiation" (Klein, 1961, p. 477). This particular quantum conjecture is thus that an oscillator of frequency $\nu$ can only emit energy in integral amounts $h \nu$, despite the fact that we would have previously assumed that there was no discrete restriction on this quantity.

An alternative reading of Planck's quantum conjecture is that he was specifically imposing a quantization condition on the phase space of a resonator when calculating its entropy. Planck himself, when discussing the ratio $P$ that results from dividing the total energy $E$ of the system by the number of energy elements, says "If the ratio is not an integer, we take for $P$ an integer in the neighbourhood (Planck, 1900/1967, 84)." The inclusion of the caveat, "in case $P$ is not an integer," seems to indicate that he considers the possibility that the energy elements are merely a convenient calculational device, and do not reflect a physical fact. Thomas Kuhn is one person who has has argued for this kind of interpretation, where it is not clear what physical significance we should attribute to this specific partition of phase space (Kuhn 1978). ${ }^{7}$ Regardless of which conjecture Planck was actually committed to, the idea of quantizing a quantity which had always previously been considered to be continuous with the parameter $h$ was used exclusively in a context considering the interaction between radiation and matter.

These two conjectures show that even the mere introduction of the idea of quantization was ambiguous in terms of its physical interpretation. The lack of a definite interpretation at least suggests that this idea was guiding research rather than acting as a hypothesis that could be straightforwardly confirmed. In the next quantum conjectures, we will see how the idea of quantization was applied beyond this context, by acting as a unifying idea to guide research in different areas.

I will first discuss Einstein's uses of the idea of quantization. The first such application appeared in Einstein's 1905 paper on light quanta, in which he suggested that the idea of quantization should be applied to electromagnetic radiation itself rather than in the interaction of radiation with matter. $\mathrm{He}$ presented the major assumption of this paper as follows:

According to the assumption considered here, when a light ray starting from a point is propagated, the energy is not continuously distributed over an ever increasing volume, but it consists of a finite number of energy quanta, localised in space, which move without being divided and which can be absorbed or emitted only as a whole. (Einstein, 1905/1967, 92)

Einstein first showed that when assuming the validity of the equipartition theorem to describe the velocities of the molecules, using Maxwell's theory to analyse the energy of resonators and gas molecules in a volume surrounded by reflecting walls leads to the prediction that the amount of radiation will continue to grow with the frequency of radiation, a result we now refer to as the ultraviolet catastrophe. Einstein saw the potential in Planck's work for solving this problem, but provided a more rigorous underpinning for the idea of quantization.

\footnotetext{
${ }^{7}$ See also Darrigol (1992) for useful discussions.
} 
Einstein used this strategy once again in his work on the specific heat of diamond. ${ }^{8}$ At the time, the observed value of this quantity was quite far from theoretical predictions. Einstein's 1907 paper was the first attempt at using a quantum conjecture in a context other than that of radiation phenomena. He proposed that the idea that energy should be considered in quantized amounts be applied to the energy of atoms of solids. Specifically, the energy of elementary structures could only assume the values $0, h \nu, 2 h \nu, \ldots$, where $h$ is Planck's constant and $\nu$ the frequency of the atom. ${ }^{9}$ This conjecture was thus not limited to the context of radiation, but any elementary structure that could be modelled as an oscillator. In fact, in this context, the energy of an oscillator has to do with its position and momentum, which seems prima facie to be unrelated to the constitution of electromagnetic radiation. This application allowed him to calculate a value for the specific heat which was much closer to the observed value. There are several features of Einstein's work in these two cases that indicate that he was not simply applying Planck's quantization conjecture, but was instead using the idea to guide his own pursuit of a theory that would explain the observed anomalies in different domains. In other words, he was supposing that unification may be operative, and employing it as a heuristic strategy.

For one thing, Einstein pointed out that certain of Planck's assumptions in the derivation of his blackbody formula were questionable in terms of their consistency, so while he was influenced by Planck's work, it is not the case that he intended his own theorizing as a mere test or extension of the theory developed by Planck (Einstein, 1906/1989). ${ }^{10}$ However, Einstein did use his criticism of Planck's assumptions to point out the need for further investigation of certain concepts that Planck was using.

A related fact is that Einstein provided a different argument to justify the appeal to the idea of quantization. While Planck was representing a blackbody as a system of vibrating resonators that could absorb and emit energy, Einstein considered a system with $n$ moving points in a given volume, in analogy with gaseous systems. Planck's appeal to Boltzmann's work in the kinetic theory of gases seemed to stem primarily from its mathematical expediency in leading to the correct radiation formula; Einstein's analogy with kinetic theory was carefully formulated so that although its applicability was limited only to domains of low-density radiation, the argument was much better grounded than Planck's. ${ }^{11}$

Finally, the differences in the domains of applicability accentuate the idea of using a unifying heuristic to guide research. Einstein's formulation of the conjecture is applicable to phenomena that Planck never considered, such as the phenomenon of fluorescence, the ionisation of gases, and the photoelectric effect. This is true despite the fact that nothing in Planck's discussions of blackbody radiation indicate that his conjecture of quantized energy was meant to be exported to other domains.

The final successful quantum conjecture I will address comes out of Niels Bohr's work on the model of the hydrogen atom. ${ }^{12}$ Bohr motivated his 1913 paper by noting that certain experimental results on $\alpha$-ray scattering seemed to support Rutherford's atomic model, but that this model comes up against conceptual and theoretical problems not encountered in alternative atomic models. One important issue was that Rutherford's model required the existence of stable states that cannot be determined based on classical electrodynamics. Furthermore, the quantities present in the Rutherford model did not provide enough information to determine a characteristic length for the radius of the atom. Bohr noted that the introduction of $h$, Planck's constant, provides this information, since its units and dimensions made it possible to calculate the length of the atom which turned out to be of the expected order of magnitude based on other experiments. Using his version of a quantum conjecture, along with his elementary atomic

\footnotetext{
8 The specific heat of a substance refers to the amount of heat required to raise a certain quantity of that substance by one degree Kelvin.

9 Einstein used a different notation which was equivalent to expressing quantities in terms of $h$.

10 The inconsistency arises from the two different ways Planck used to calculate the equilibrium entropy of a blackbody. The first assumes that the energy of the resonator takes on integral multiples of an undetermined amount $\epsilon$. The other relies on a calculation using Maxwell's theory of electricity, which are expressions of periodic functions that do not restrict their possible values to discrete ones.

11 See Norton (2006) for a reconstruction of the reasoning.

12 See Heilbron and Kuhn (1969) for a detailed treatment of this episode.
} 
model, Bohr was able to account for the Balmer formula, which describes the discrete spectral lines observed when hydrogen gas is heated.

Although Bohr explicitly referenced Planck's quantization conjecture, Bohr's interpretation of quantization is a novel one. Several quotes from Bohr emphasize this fact. For instance,

It is readily seen that there can be no question of a direct application of Planck's theory. This theory is concerned with the emission and absorption of energy in a system of electrical particles, which oscillate with a given frequency per second, dependent only on the nature of the system and independent of the amount of energy contained in the system. (Bohr, 1922, 10)

This is in contrast to an atomic system, where the frequency depends on the energy of the system. Therefore, despite the reference to the emission of discrete amounts of energy, this was not a straightforward application of Planck's idea. Therefore, we see that Bohr, like Einstein, was using a unifying strategy to further pursue his inquiries in the domain of atomic structure. Again, the value of unification is as a heuristic rather than as a feature that justified the hypothesis.

\section{Discussion and implications}

\subsection{A heuristic strategy}

In the previous section, I gave a brief overview of how the idea of quantization was acting as a unifying idea that scientists such as Planck, Einstein, and Bohr used to pursue work in different domains of physics. In this section, I will discuss the significance of recognizing that unification guides theory pursuit rather than simply contributes to the support for a theory.

The first point is simply that this provides an accurate description of one element in the process of scientific theory development during a crucial historical episode. It makes sense of the role that unification played in scientists' reasoning at the time, namely as a strategy for approaching various empirical problems. We saw that by employing such a strategy, both Einstein and Bohr were able to account for phenomena that previously had no explanation in certain domains, despite the fact that there was no overarching consistent theory that scientists were trying to apply in different contexts. This heuristic attitude is displayed in the final section of Einstein's (1909), where he sketches a possible interpretation of the meaning of light quanta in which the energy of the electromagnetic field is localized in singular points. After this description, he goes on to say, "I am sure it need not be particularly emphasized that no importance should be attached to such a picture as long as it has not led to an exact theory" (Einstein, 1909/1989, 394).

Indeed, it does not even seem to be the case that finding an overarching theory was the primary motivation for work in the various domains. While this might be relatively self-evident, it is worth noting that this is the opposite of what we might think would be the case if the goal were, for instance, to find unifying theories because unifying explanations are a crucial characteristic of theories. Instead, it seemed that the primary motivation was to solve existing problems in each scientific domain. Scientists accomplished this by using the idea of quantization as a heuristic tool to account for problematic phenomena. Thus, during this early stage of theory development, Bohr, for instance, did not claim that the feature of unification made the idea of quantization more plausible; instead, he used the idea in a unifying way as a strategy for developing a new theory of the hydrogen atom. By turning our attention from the justificatory aspect of unification, we obtain a better understanding of the process of theory development. The historical analysis thus contributes to the characterization of successful reasoning patterns that occur in such early stages.

Perhaps more importantly for our purposes, this historical example can help us to understand the way in which unification, understood as a heuristic strategy, can contribute directly to the development of a 
theory. In our particular case, we can see how such a strategy helped determine both the scope and the content of an eventual quantum theory.

My focus will be on the former, but I will provide a brief example of how a unifying strategy helps to determine the content of a quantum theory. I refer to the fact that the experiments that were performed in each of the different domains can be interpreted as contributing to the determination of the value of Planck's constant, which is a crucial part of the theory of quantum mechanics that was subsequently developed. ${ }^{13}$ The basic idea is that the numerical value of a theoretical parameter is an important element of a theory, and by drawing on the fact that experiments in different domains provided multiple measurements of Planck's constant we see how part of the quantum theory was determined in this way. While the notion of agreeing measurements is hardly novel, I would emphasize here that the determination of the value of Planck's constant was not simply a confirmational exercise, but an actual contribution to the content of quantum theory, broadly speaking. Thus, we can see the importance of using a strategy of unification for the articulation of a theory, apart from the contribution of that particular feature to the resulting theory's evidential support.

Equally important in the context of pursuing a new theory is that of determining its scope, where I use this word to refer to the theory's domain of applicability. One of the questions that should naturally arise in the introduction of a quantization parameter is whether it is simply a feature of the particular system under study, such as the interaction between matter and radiation in Planck's work, or whether it is a result of a more general condition. Any individual application of the idea of quantization could be considered merely as a way of trying to recover the observations of the phenomenon in question. This is demonstrated by the fact that quantization itself could be attributed to various possible physical causes. Indeed, we can understand Planck's original introduction of the idea in just these terms.

However, the success of using a quantum postulate in such a wide range of domains showed that there must be something about the underlying description of the systems that was common. After all, it might have been possible to account for the spectrum of blackbody radiation in terms of some mechanism in a resonator that would render its emission of electromagnetic radiation a discrete process. Nevertheless, such a mechanism would be specific to the situation, in which energy is directly related to the frequency of electromagnetic radiation. Such a mechanism would not be applicable in the context of an oscillator whose energy depends on position and momentum, such as those used to model a diamond. The increasingly broad applicability of the notion of quantization began to reduce the probability that previous continuous descriptions of quantities could be wholly accurate. Thus, the fact that quantization was applicable in these disparate contexts allowed scientists to eliminate the possibility that certain specific physical causes were responsible for the observed behaviour. The 'indefinite' nature of the postulate is thus actually an indication of how universal it is, and this universality made it increasingly clear that some fundamental aspect of previous descriptions of physical systems had to change. These results provided evidence that any future theory must address not only any particular dynamical aspects of physical setups, but the fundamental kinematical descriptions of systems at very small scales.

The idea that using unification as a heuristic strategy contributes to the development of a theory differs from the judgement that unifying hypotheses are better supported than non-unifying hypotheses. It also differs from the project of evaluating a theory as an explanation of all the given phenomena, though I certainly would not deny that this can often be valuable. Again, the crucial issue here is not how diverse various sources of evidence for a given hypothesis happen to be, but rather the value for the theory itself in employing such a strategy. The assumption is not that unification per se is good; instead, the observation is that the adoption of unification as a heuristic is a good strategy for making progress on a theory.

One might be tempted to say that using a unifying strategy is useful simply because of its possible fruitfulness, that is, its potential ability to successfully solve problems in different domains. Indeed, it is doubtful that anyone would deny that this is a crucial element of theory development. However, I claim

\footnotetext{
13 See Kao (2015) for discussion of this idea.
} 
that it is also important to identify a related but different result: such an account brings out the importance of applying a unifying hypothesis in ultimately unsuccessful contexts. For instance, consider the following work by Arnold Sommerfeld, a prominent contributor to the development of quantum theory. He noted in his report at the first Solvay Congress that the crucial parameter $h$ was a quantum of action, not of energy. He thus hypothesized that a quantum condition should be imposed not on energy itself, such as in Einstein's suggestion of light quanta, but on the processes governing energy changes over time in a given process. ${ }^{14}$ This led him to formulate a quantum postulate in terms of the Lagrangian of a system, $\int_{0}^{\tau} L d t=h / 2 \pi$, where $L$ is the Lagrangian (kinetic minus potential energy), and $\tau$ represents the duration of the process involving an energy exchange $(1912,316)$. He and Peter Debye later used this postulate to predict a relation between $\tau$ and $E$, the electric amplitude, in the context of radiation absorption and emission (1913). However, no such relation was found between these two quantities.

If we focus on how quantum theory was being developed, we can see that such an attempt at applying the postulate actually helped to delimit its scope. Namely, the failure of this application pointed to the idea that quantization was not necessarily related to the dynamical process of energy exchange between radiation and matter. On the other hand, if we focus on unification solely in terms of its contribution to justification, it is difficult to account for the value of such unsuccessful applications of unifying ideas, since they are essentially irrelevant to the support for a theory. Of course, one might reply that if such a prediction had been borne out, this would have provided some reason to think that more applications of quantization might be found in the examination of dynamical processes, and so the value of this line of inquiry still lies in its potential contribution. However, I would argue that it is more accurate to recognize the positive value of the negative result: by combining it with successful results such as the quantization of the phase space of an oscillator, it became increasingly clear that quantization should apply to descriptions of systems rather than temporal processes.

\subsection{Relation to robustness reasoning}

The focus on unification as a heuristic strategy parallels certain elements of a related type of reasoning, namely that found in robustness analysis. While there are several ways of describing robustness reasoning, we may express the common thread as follows: a hypothesis is deemed more plausible due to the fact that it is supported by multiple lines of argument (where "hypothesis" may refer to a prediction, a description of a system, or another aspect of a theory). Such hypotheses might include statements about particular theoretical parameters supported by agreeing measurements in diverse experiments, the existence of a general phenomenon that may manifest in several different target systems, or predictions about a specific system that is being represented by multiple models. ${ }^{15}$ First, it is worth being explicit about how unification as described in this paper differs from robustness analysis. While the former is meant to be a strategy that guides the articulation of a hypothesis that ultimately connects multiple domains, the latter is focused on the way in which multiple lines of argument support a given hypothesis. Nevertheless, the two share some interesting common features.

One such feature is simply their focus on the process of developing a theory rather than evaluating it. For instance, Trizio (2012) and Nederbragt (2012) both describe the role of robustness analysis in cases of ongoing research; Trizio by describing a particular case where robust results are an explicit goal when conducting experiments, and Nederbragt by describing how robustness reasoning can be used to confirm results of local hypotheses within broader theories. Both of these discussions are firmly situated within the

\footnotetext{
14 "Nous ferons, par suite, l'hypothèse suivante. Les propriétés générales de toutes les molécules ou atomes qui déterminent les phénomènes de rayonnement ne consistent pas dans l'intervention d'éléments particuliers d'énergie, mais en ceci, que la manière dont se produisent les échanges d'énergie dans un temps plus ou moins long est dominée par une loi universelle" (Sommerfeld, 1912, 314).

15 See Schickore and Coko (2013) for an overview and further references.
} 
context of articulating a theory rather than evaluating a result. Weisberg (2006) emphasizes a similar point in his discussion of robustness analysis in the context of phenomena studied by population biologists. He characterizes robustness analysis as a process by which scientists can identify robust features and robust theorems. In other words, it is a method of analysis that can help to actually articulate a hypothesis or theory.

Weisberg also contrasts the types of systems he is discussing — primarily those studied by population biologists - with physical systems described by fundamental physical theories. He points out that in the latter, the effect of certain idealizations found in a model of the system can be estimated, whereas "in the study of many complex systems, ... such a theory is unavailable" $(2006,713)$. While it is clear that Weisberg is referring to relatively simple examples such as the analysis of a mass sliding down an inclined plane, it is worth noting that at the stage of developing fundamental physical theories, the same problems are inherent, and an analogous strategy needs to be employed.

The foregoing discussion might lead one to question the wisdom or utility of taking unification to be a heuristic strategy. After all, one might think that doing so requires either that we have a preexisting commitment to the unity of science, or that we have some reason to think that unified theories are more likely to be true. Indeed, Weisberg himself argues that theorems arrived at through robustness analysis are more likely to be true. I will treat these two considerations in turn.

First, I will address the idea that considering unification to be a useful heuristic strategy presupposes that science is an essentially unifying enterprise. Of course, the "unity of science" thesis has been discussed extensively in philosophy, and it is not the goal of this paper to argue either for or against such a thesis. Instead, I will note that by focusing on the process of developing a theory, we need not be committed to the idea that the final theory will definitely unify all of the phenomena being investigated; a perfectly legitimate outcome of using a unifying heuristic is a result in which one concludes that there is no unifying element between multiple phenomena. In such a case, knowledge is gained about the domain of applicability of a hypothesis. Furthermore, I take it to be an asset of this account that developed theories may be unifying in different ways. While I have discussed this case in the context of early quantum theory in which multiple domains were unified by their incorporation of a quantization parameter into their physical description, there exist a multitude of ways of following such a strategy. For instance, Myrvold (2017) and Nathan (2017) provide different interpretations of unification. Furthermore, Morrison (2000) argues by examining several historical cases that there is no unified account of theoretical unification. ${ }^{16}$ This is not problematic; it simply indicates that the application of unification as a heuristic strategy will likely be different in different disciplines.

The second point expresses the concern that the characterization of unification as a heuristic strategy is parasitic on the notion of justification. In other words, perhaps unification is only useful as a guiding strategy for theory pursuit because such hypotheses are subsequently better supported by the evidence, where such support can be understood in different ways. Thus, the claim that we should understand unification as a heuristic strategy does not add much to our understanding. However, such a claim is rather beside the point. I certainly do not deny the value of analyses of how the feature of unification provides support for a given theory. Indeed, I accept the idea that unification contributes to the support for a theory in many cases. Despite this fact, considering unification in a heuristic context allows us to see how such a strategy contributes directly to the articulation of a newly developing theory by helping to determine its content and scope. This is true even apart from the support the resulting theory may receive from multiple contexts, since the unification contributes to the construction of the hypothesis itself.

Indeed, we see that this aspect of unification plays a role prior to the analysis of the support it provides for a hypothesis, insofar as it is a strategy that can be used to help construct a theory before we reach the point of evaluating that theory in terms of it or by other means. We need not think that the theory that applies to more phenomena is more likely to be true; we simply recognise that in order to arrive

\footnotetext{
16 She also provides a thorough argument decoupling unification from both truth and explanation.
} 
at a theory, we must adopt certain strategies. Given the case of the quantum postulate, we saw that the strategy of considering the quantum postulate to be possibly applicable in multiple contexts helped to actually constrain how the postulate should be understood, and was thus crucial for the development of a further quantum theory. By focusing solely on unification in the logical context of justifying a theory, we ignore a crucial element of the developmental process. An understanding of unification in this context provides a fuller picture of scientific practice.

\section{Conclusion}

In this article, I have argued that we must consider the role of the theoretical feature of unification in the context of developing a theory, and not only in the context of providing support for that theory. I have argued that unification can act as a general heuristic strategy when developing a new theory, and supported this characterization by showing how such a strategy was operative in the early stages of the development of quantum theory. This characterization is distinct from many current discussions of unification since the emphasis is on how it can contribute to the preliminary articulation of a theory.

This conception has several favourable features. First, it provides what I claim is a faithful description of one of the ways scientists do in fact approach certain problems in science. Second, I have argued that such an analysis allows us to better understand how a theory is developed by determining the domain of applicability of certain hypotheses. Furthermore, this analysis provides an account of the positive contribution of failed lines of research, whose value is not evident when discussing unification in the context of evidential support. While this is not an argument against the value of these other conceptions of unification, it is meant to draw attention to the fact that we cannot ignore the heuristic role if we wish to properly describe and understand how certain theories are developed.

\section{References}

Bohr N (1913) On the constitution of atoms and molecules, part I. Philosophical Magazine 26:1-25

Bohr N (1922) The Theory of Spectra and Atomic Constitution: Three Essays. Cambridge University Press, Great Britain

Darrigol O (1992) From c-Numbers to q-Numbers: The Classical Analogy in the History of Quantum Theory. University of California Press, Berkeley, retrieved from http://ark.cdlib.org/ark:/13030/ft4t1nb2gv/

Debye P, Sommerfeld A (1913) Theorie des lichtelektrischen Effektes vom Standpunkt des Wirkungsquantums. Annalen der Physik (10):873-930

Einstein A (1905) Ueber einen die Erzeugung und Verwandlung des Lichtes betreffenden heuristischen Gesichtspunkt. Annalen der Physik 17:132-148, translated by D. ter Haar in The Old Quantum Theory 1967, pp. 91-107

Einstein A (1905/1967) On a heuristic point of view about the creation and conversion of light. In: The Old Quantum Theory: Selected Readings in Physics, Pergamon Press, Great Britain, original work published 1905

Einstein A (1906/1989) On the theory of light production and light absorption. In: The Collected Papers of Albert Einstein, Volume 2: The Swiss Years: Writings, 1900-1909 (English translation supplement), pp 192-199, original work published 1906

Einstein A (1907) Die Plancksche Theorie der Strahlung und die Theorie der spezifischen Waerme. Annalen der Physik 22:180-190

Einstein A (1909) Über die Entwickelung unserer Anschauungen über das Wesen und die Konstitution der Strahlung. Deutsche Physikalische Gesellschaft 7:482-500 
Einstein A (1909/1989) On the development of our views concerning the nature and constitution of radiation. In: The Collected Papers of Albert Einstein, Volume 2: The Swiss Years: Writings, 1900-1909 (English translation supplement), pp 379-394, original work published 1909

Forster MR (1988) Unification, explanation, and the composition of causes in Newtonian Mechanics. Studies in History and Philosophy of Science 19:55-101

Forster MR (2010) Miraculous consilience of quantum mechanics. In: Eells E, Fetzer JH (eds) The Place of Probability in Science: In Honor of Ellery Eells (1953-2006), Springer, pp 201-228

Harper W (2002) Newton's argument for universal gravitation. In: Cohen IB, Smith GE (eds) The Cambridge Companion to Newton, Cambridge University Press, Cambridge, pp 174-201

Heilbron JL, Kuhn TS (1969) The genesis of the Bohr atom. Historical Studies in the Physical Sciences $1: 211-290$

Helgeson C (2013) The confirmational significance of agreeing measurements. Philosophy of Science 80:721-732

Janssen M (2002) COI stories: Explanation and evidence in the history of science. Perspectives on Science 10(4):457-522

Kao M (2015) Unification and the quantum hypothesis in 1900-1913. Philosophy of Science 82:12001210

Kitcher P (1981) Explanatory unification 48(4):507-531

Kitcher P (1989) Explanatory unification and the causal structure of the world. In: Kitcher P, Salmon WC (eds) Scientific Explanation: Minnesota Studies in the Philosophy of Science, Volume XIII, University of Minnesota Press, Minneapolis, pp 410-505

Klein MJ (1961) Max Planck and the beginnings of the quantum theory. Archive for History of Exact Sciences 1(5):459-479

Kuhn TS (1978) Black-body Theory and the Quantum Discontinuity: 1894-1912. Oxford University Press, Inc., New York, United States of America

Kuorikoski J, Marchionni C (2016) Evidential diversity and the triangulation of phenomena. Philosophy of Science 83:227-247

McGrew T (2003) Confirmation, heuristics, and explanatory reasoning. The British Journal for the Philosophy of Science 54(4):553-567

Miyake T (2015) Underdetermination and decomposition in kepler's Astronomia Nova. Studies in History and PHilosophy of Science 50:20-27

Morrison M (2000) Unifying Scientific Theories: Physical Concepts and Mathematical Structures. Cambridge University Press, New York

Myrvold WC (2003) A Bayesian account of the virtue of unification. Philosophy of Science 70:399-423

Myrvold WC (2017) On the evidential import of unification. Philosophy of Science 84:92-114

Nathan MJ (2017) Unificatory explanation. British Journal for the Philosophy of Science 68:163-186

Nederbragt H (2012) Multiple derivability and the reliability and stabilization of theories. In: Characterizing the Robustness of Science: After the Practice Turn in Philosophy of Science, Springer, pp 121-145

Norton JD (2006) Atoms, entropy, quanta: Einstein's miraculous argument of 1905. Studies in History and Philosophy of Modern Physics 37:70-100

Planck M (1900) Zur Theorie des Gesetzes der Energieverteilung im Normalspectrum. Verhandlungen der Deutschen Physikalischen Gesellschaft 2:237-245

Planck M (1900/1967) On the theory of the energy distribution law in the normal spectrum. In: The Old Quantum Theory, pp 82-90, original work published 1900

Planck M (1901) Über das Gesetz der Energieverteilung im Normalspectrum. Annalen der Physik 4:553563, translated by Koji Ando

Schickore J, Coko K (2013) Using multiple means of determination. International Studies in the Philosophy of Science 27(3):295-313 
Schupbach JN (2005) On a Bayesian analysis of the virtue of unification. Philosophy of Science 72(4):594-607

Schupbach JN (Forthcoming) Robustness analysis as explanatory reasoning. British Journal for the Philosophy of Science Advance Access, doi:10.1093/bjps/axw008

Sommerfeld A (1912) Application de la théorie de l'élément d'action aux phénomènes moléculaires non périodiques. In: Langevin P, de Broglie M (eds) La Théorie du Rayonnement et les Quanta : Rapports et discussions de la réunion tenue à Bruxelles, du 30 octobre au 3 novembre 1911, pp 313-372

Spohn W (1994) On Reichenbach's principle of the common cause. In: Logic, Language, and the Structure of Scientific Theories: Proceedings of the Carnap-Reichenbach Centennial, University of Konstanz, 21-24 May 1991, University of Pittsburgh Press, Pittsburgh, pp 211-235

Trizio E (2012) Achieving robustness to confirm controversial hypotheses: A case study in cell biology. In: Characterizing the Robustness of Science: After the Practice Turn in Philosophy of Science, Springer, pp 105-120

Weisberg M (2006) Robustness analysis. Philosophy of Science 73(5):730-742, proceedings of the 2004 Biennial Meeting of the Philosophy of Science Association, Part II: Symposia

Whewell W (1989) Theory of Scientific Method. Hackett Publishing Company, United States of America, ed. Robert E. Butts 